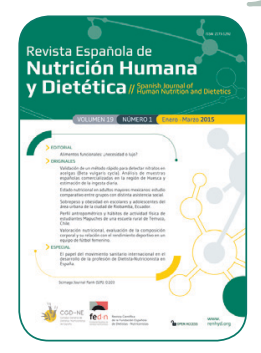

\title{
EDITORIAL
}

\section{Formulación de Políticas Nutricionales informadas por la Evidencia}

\author{
Eduard Baladia $^{a, b, *}$, Rodrigo Martínez-Rodríguez ${ }^{a}$, José Miguel Martínez- Sanz ${ }^{b, c}$,Aurora Norte \\ Navarro $^{\text {b,c }}$, Rocío Ortiz- Moncada ${ }^{b, c}$
}

a Centro de Análisis de la Evidencia Científica de la Fundación Española de Dietistas-Nutricionistas (CAEC-FEDN), España.

b Grupo de Investigación en Alimentación y Nutrición (ALINUT), Universidad de Alicante, España.

C Gabinete de Alimentación y Nutrición de la Universidad de Alicante (ALINUA), España.

*baladia@ebaladia.es

Recibido el 23 de junio de 2015; aceptado el 26 de junio de 2015.

\section{CITA}

Baladia E, Martínez-Rodríguez R, Martínez-Sanz JM, Norte Navarro A, Ortiz-Moncada R. Formulación de Políticas Nutricionales informadas por la Evidencia. Rev Esp Nutr Hum Diet. 2015; 19(2): 56 - 57. D0I: 10.14306/ renhyd.19.2.162

La implementación de políticas nutricionales, debería ser uno de los últimos pasos seguidos en el proceso de la toma de decisiones políticas ${ }^{1}$ y, seguramente, el primer paso debería ser asegurar que todos los agentes implicados estén de acuerdo en que la formulación de políticas nutricionales debe estar siempre informada por la evidencia científica y teniendo en consideración las adecuadas condiciones de equidad. Sin embargo, desgraciadamente sabemos que no siempre es así?.

En 2009 se publicaron una serie de 19 artículos $^{1,3-20}$ en la revista BioMed Central en los que se explica, desde qué es la formulación de políticas en salud informadas en la evidencia, hasta cómo buscar, interpretar y usar la evidencia científica; qué hacer en caso de no haber suficiente evidencia; cómo evaluar los pros y contras de una política; cómo planear su implementación teniendo en cuenta el uso de recursos y coste de la misma; y cómo monitorizar y evaluar las políticas puestas en marcha.

Como en toda toma de decisiones basada en la evidencia, estas guías no se han elaborado para sustituir la figura de las personas encargadas de tomar dichas decisiones, sino para ayudar a que tanto los responsables de la toma de decisiones sobre políticas y programas de salud, como quienes los respaldan (inclusive organizaciones de científicos), tomen consciencia de la necesidad de que las decisiones se realicen siempre teniendo en cuenta la mejor evidencia de investigación disponible ${ }^{1}$.

Entre las reflexiones a las que lleva la lectura de estos artículos, a los autores de estas líneas les ha parecido especialmente interesante el cuestionamiento de qué estrategias deberían usarse para evitar posibles presiones de grupos de poder financieros sobre una decisión de política de salud. En este sentido, la descripción de una metodología sistemática y transparente previa a la toma de decisiones y fácilmente accesible por todos, o la participación de grupos de expertos que usan metodologías basadas en la evidencia, como por ejemplo, los grupos elaboradores de Guías de Práctica Clínica Basadas en la Evidencia (GuíaSalud en España) o las Agencias de Evaluación de Tecnologías Sanitarias, pueden ayudar a manejar las tensiones y conflictos de interés que puedan generarse entre los grupos de poder económico y las decisiones políticas tomadas ${ }^{4}$.

Sin embargo, los sistemas basados en metodologías sistemáticas y transparentes que busquen el apoyo de partes proactivas (generación de la evidencia por parte de terceros independientes) que generen resúmenes de políticas ${ }^{15}$ y partes reactivas (partes financieras implicadas) a través de un 
diálogo de políticas ${ }^{16}$, podría desajustar mucho el tiempo en el que se requiere la toma de decisiones. En este sentido, ha Ilamado la atención de los autores de este escrito, la dedicación de una sección en la revista BioMed Central a la interesante cuestión de cómo priorizar las decisiones políticas en función del tiempo en el que se requiere dicha toma de decisiones, pudiéndose definir estrategias de enfoque rápido aunque menos consistente y otras de enfoque más lento pero más potente 5 .

Asimismo, parece ser que los autores de esta serie de artículos hayan pensado en todas las posibles barreras que las personas relacionadas con la toma de decisiones en políticas de salud pudieran tener para llevar a cabo su importante tarea, elaborando una herramienta útil y de fácil entendimiento para superar cada una de dichas dificultades. Los artículos revisan a fondo cómo usar la investigación para definir adecuadamente un problema, cómo establecer un marco de opciones posibles para abordar dicho problema, cómo evaluar la aplicabilidad de la evidencia, cómo tener en cuenta la equidad, cómo evaluar los costes de su implementación o incluso cómo monitorizar y evaluar las políticas implementadas.

Ha llegado el momento de reflexionar sobre estos y otros muchos temas que plantean estos magníficos 19 artículos ${ }^{1,3-20}$, y ha llegado el momento de pedir que todas aquellas personas relacionadas (ya sea de forma directa o indirecta) con la toma de decisiones en políticas nutricionales tomen consciencia de la necesidad urgente de que dicha toma de decisiones sea bien informada a través de la evidencia científica, y hacerles saber que disponen de 18 herramientas estupendas (Ilamadas SUPPORT tools) para ayudarles en la implementación de un sistema que conduzca a la posibilidad de tener un sistema de gestión de salud más eficiente.

\section{BIBLIOGRAFÍA}

1. Lavis JN, Oxman AD, Lewin S, Fretheim A. SUPPORT Tools for evidence-informed health Policymaking (STP). Health Res Policy Syst BioMed Cent. 2009; 7 Suppl 1: 1.

2. Marques-Lopes I. Efecto de las desigualdades sociales en la prevalencia de la obesidad. Rev Esp Nutr Humana Dietética. 30 de junio de 2013; 17(2): 45-6.

3. Oxman $A D$, Lavis JN, Lewin $S$, Fretheim A. SUPPORT Tools for evidence-informed health Policymaking (STP) 1: What is evidence-informed policymaking? Health Res Policy Syst BioMed Cent. 2009; 7 Suppl 1: S1.

4. Oxman AD, Vandvik PO, Lavis JN, Fretheim A, Lewin S. SUPPORT Tools for evidence-informed health Policymaking (STP) 2: Improving how your organisation supports the use of research evidence to inform policymaking. Health Res Policy Syst BioMed Cent. 2009; 7 Suppl 1: S2.

5. Lavis JN, Oxman AD, Lewin S, Fretheim A. SUPPORT Tools for evidence-informed health Policymaking (STP) 3: Setting priorities for supporting evidence-informed policymaking. Health Res Policy Syst BioMed Cent. 2009; 7 Suppl 1: S3.

6. Lavis JN, Wilson MG, Oxman AD, Lewin S, Fretheim A. SUPPORT Tools for evidence-informed health Policymaking (STP) 4: Using research evidence to clarify a problem. Health Res Policy Syst BioMed Cent. 2009; 7 Suppl 1: S4.
7. Lavis JN, Wilson MG, Oxman AD, Grimshaw J, Lewin S, Fretheim A. SUPPORT Tools for evidence-informed health Policymaking (STP) 5: Using research evidence to frame options to address a problem. Health Res Policy Syst BioMed Cent. 2009; 7 Suppl 1: S5.

8. Fretheim A, Munabi-Babigumira S, Oxman AD, Lavis JN, Lewin S. SUPPORT tools for evidence-informed policymaking in health 6: Using research evidence to address how an option will be implemented. Health Res Policy Syst BioMed Cent. 2009; 7 Suppl 1: S6.

9. Lavis JN, Oxman AD, Grimshaw J, Johansen M, Boyko JA, Lewin $S$, et al. SUPPORT Tools for evidence-informed health Policymaking (STP) 7: Finding systematic reviews. Health Res Policy Syst BioMed Cent. 2009; 7 Suppl 1: S7.

10. Lewin $S$, Oxman AD, Lavis JN, Fretheim A. SUPPORT Tools for evidence-informed health Policymaking (STP) 8: Deciding how much confidence to place in a systematic review. Health Res Policy Syst BioMed Cent. 2009; 7 Suppl 1: S8.

11. Lavis JN, Oxman AD, Souza NM, Lewin S, Gruen RL, Fretheim A. SUPPORT Tools for evidence-informed health Policymaking (STP) 9: Assessing the applicability of the findings of a systematic review. Health Res Policy Syst BioMed Cent. 2009; 7 Suppl 1: S9.

12. Oxman AD, Lavis JN, Lewin S, Fretheim A. SUPPORT Tools for evidence-informed health Policymaking (STP) 10: Taking equity into consideration when assessing the findings of a systematic review. Health Res Policy Syst BioMed Cent. 2009; 7 Suppl 1: S10.

13. Lewin $S$, Oxman AD, Lavis JN, Fretheim A, Garcia Marti $S$, Munabi-Babigumira S. SUPPORT tools for evidence-informed policymaking in health 11: Finding and using evidence about local conditions. Health Res Policy Syst BioMed Cent. 2009; 7 Suppl 1: S11.

14. Oxman AD, Fretheim A, Lavis JN, Lewin S. SUPPORT Tools for evidence-informed health Policymaking (STP) 12: Finding and using research evidence about resource use and costs. Health Res Policy Syst BioMed Cent. 2009; 7 Suppl 1: S12.

15. Lavis JN, Permanand G, Oxman AD, Lewin S, Fretheim A. SUPPORT Tools for evidence-informed health Policymaking (STP) 13: Preparing and using policy briefs to support evidenceinformed policymaking. Health Res Policy Syst BioMed Cent. 2009; 7 Suppl 1: S13.

16. Lavis JN, Boyko JA, Oxman AD, Lewin S, Fretheim A. SUPPORT Tools for evidence-informed health Policymaking (STP) 14: Organising and using policy dialogues to support evidenceinformed policymaking. Health Res Policy Syst BioMed Cent. 2009; 7 Suppl 1: S14.

17. Oxman AD, Lewin $S$, Lavis JN, Fretheim A. SUPPORT Tools for evidence-informed health Policymaking (STP) 15: Engaging the public in evidence-informed policymaking. Health Res Policy Syst BioMed Cent. 2009; 7 Suppl 1: S15.

18. Oxman AD, Lavis JN, Fretheim A, Lewin S. SUPPORT Tools for evidence-informed health Policymaking (STP) 16: Using research evidence in balancing the pros and cons of policies. Health Res Policy Syst BioMed Cent. 2009; 7 Suppl 1: S16.

19. Oxman AD, Lavis JN, Fretheim A, Lewin S. SUPPORT Tools for evidence-informed health Policymaking (STP) 17: Dealing with insufficient research evidence. Health Res Policy Syst BioMed Cent. 2009; 7 Suppl 1: S17.

20. Fretheim $A$, Oxman $A D$, Lavis $J N$, Lewin S. SUPPORT tools for evidence-informed policymaking in health 18: Planning monitoring and evaluation of policies. Health Res Policy Syst BioMed Cent. 2009; 7 Suppl 1: S18. 